\title{
Secondary Increase of Lactate Levels in Asphyxiated Newborns during Hypothermia Treatment: Reflect of Suboptimal Hemodynamics (A Case Series and Review of the Literature)
}

\author{
Asim Al Balushi, MD ${ }^{1}$ Marie-Pier Guilbault, MD ${ }^{1} \quad$ Pia Wintermark, MD² \\ ${ }^{1}$ Department of Pediatrics, Montreal Children's Hospital, McGill \\ University, Montreal, Canada \\ ${ }^{2}$ Division of Newborn Medicine, Department of Pediatrics, Montreal \\ Children's Hospital, McGill University, Montreal, Canada \\ Address for correspondence Pia Wintermark, MD, Division of \\ Newborn Medicine, Montreal Children's Hospital, Research Institute of \\ the McGill University Health Centre, 1001 Boulevard Décarie, Site Glen, \\ Block E, EM0.3244, Montréal, Quebec H4A 3J1, Canada \\ (e-mail: pia.wintermark@bluemail.ch).
}

Am J Perinatol Rep 2016;6:e48-e58.

\begin{abstract}
Objective To evaluate whether a secondary increase of serum lactate levels in asphyxiated newborns during hypothermia treatment may reflect suboptimal dynamics.

Methods Retrospective case series and review of the literature. We present the clinical course of four asphyxiated newborns treated with hypothermia who presented with hypotension requiring inotropic support, and who displayed a secondary increase of serum lactate levels during hypothermia treatment. Serial serum lactate levels are correlated with blood pressure and inotropic support within the first 96 hours of life. Results Lactate levels initially decreased in the four patients. However, each of them started to present lower blood pressure, and lactate levels started to increase again. Inotropic support was started to raise blood pressure. The introduction of an epinephrine drip consistently worsened the increase of lactate levels in these newborns, whereas dopamine and dobutamine enabled the clearance of lactate in addition to raising the

\section{Keywords}

- birth asphyxia

- hemodynamics

- lactate

- neonatal encephalopathy

- newborn brain blood pressure. Rewarming was associated with hemodynamics perturbations (a decrease of blood pressure and/or an increase of lactate levels) in the three newborns who survived.

Conclusions Lactate levels during the first 4 days of life should be followed as a potential marker for suboptimal hemodynamic status in term asphyxiated newborns treated with hypothermia, for whom the maintenance of homeostasis during hypothermia treatment is of utmost importance to alleviate brain injury.
\end{abstract}

Hypotension is a common finding in asphyxiated newborns treated with hypothermia. The available systemic database review of asphyxiated newborns treated with hypothermia reports an incidence of hypotension in $51 \%$ of the treated newborns. ${ }^{1}$ Asphyxia and hypothermia are known to induce hypotension and decreased cardiac contractility and output. $^{2,3}$ Asphyxia causes myocardial dysfunction by directly decreasing blood flow to the heart, which leads to myocardial ischemia, ${ }^{4}$ as well as a reduction of cardiac output and a decreased contractility that can worsen if hypotension is received

April 11, 2015

accepted after revision

September 2, 2015

published online

October 28, 2015
DOI http://dx.doi.org/

10.1055/s-0035-1565921.

ISSN 2157-7005.
Copyright $\odot 2016$ by Thieme Medical

Publishers, Inc., 333 Seventh Avenue,

New York, NY 10001, USA.

Tel: +1(212) 584-4662.
License terms

(요 (1) $\Theta \circledast$ 
present. ${ }^{3,5}$ Therapeutic hypothermia induces physiological cardiovascular adaptations in asphyxiated newborns that lead to decrease in the myocardial damage. ${ }^{6,7}$ During hypothermia treatment, the heart rate decreases linearly as the temperature drops; this phenomenon is secondary to the slow diastolic repolarization in the sinoatrial node. ${ }^{8}$ Both cardiac output and stroke volume are reduced during hypothermia treatment. ${ }^{5}$ In addition, hypothermia has been shown to induce an initial surge of catecholamines followed by a fall in serum catecholamines, which explains why exogenous catecholamine support might be needed during hypothermia treatment. ${ }^{9}$ During hypothermia, the response to inotropic agents has been shown to shift from a $\beta$-adrenergic response to a more $\alpha$-adrenergic response. ${ }^{10,11}$ Currently, various inotropic agents are available to treat hypotension in newborns; however, important variations in practice by different institutions and by different neonatologists in the use of inotropic support have been reported. ${ }^{12}$ No clear consensus exists as to which amines or group of amines are the most appropriate to use for asphyxiated newborns treated with hypothermia.

Another major pathological process induced by asphyxia is the impairment of cerebral blood flow autoregulation, especially during the hypotension episodes as shown by the animal models of asphyxia. ${ }^{13}$ This process leads to brain injury during asphyxia. ${ }^{13}$ Since hypotension may have the potential to compromise further brain perfusion during the first days of life while the asphyxiated newborn is treated with hypothermia, the hypotension may worsen the brain injury in asphyxiated newborns. The current literature is limited regarding the impact of hypotension and its management of the hemodynamics in asphyxiated newborns treated with hypothermia. Moreover, studies that assess the impact of hypotension on mortality and the development of brain injury are lacking. Also, in many institutions, functional echocardiography is not available to guide and monitor the use of inotropic support.

Lactate levels are widely used in asphyxiated newborns as a plasma marker for tissue perfusion with possible correlation to hypoxia- and ischemia-related injury. ${ }^{14,15}$ Under normal physiological and aerobic conditions, glucose is converted to pyruvate through the glycolytic pathway, and subsequently pyruvate is converted by the pyruvate dehydrogenase enzyme to acetyl coenzyme A, which enters the Krebs cycle to yield energy in the form of adenosine triphosphate through mitochondrial oxidative phosphorylation. However, in the absence of oxygen, pyruvate is converted to lactate through the lactate dehydrogenase enzyme. Hypoxia-ischemia blocks the oxidative phosphorylation in the mitochondria, leading to reduced production of adenosine triphosphate, energy failure, and the accumulation of pyruvate, with the preferred formation of lactate through the initiation of an anaerobic metabolism. ${ }^{16,17}$ The effect of hypothermia on lactate production is unknown. ${ }^{6}$ In addition, during hypothermia, lactate production, even without metabolic acidosis, may signal hypoxia at the tissue level and a worsening of organ perfusion. ${ }^{6}$ Lactate production could also be due to factors that are unrelated to hypoxia or ischemia, such as hypoglyce- mia, posthyperglycemic state, and medications such as epinephrine and vasopressin. ${ }^{18}$ In the brain, increased lactate levels measured by magnetic resonance spectroscopy have been correlated with poor outcome in asphyxiated newborns. ${ }^{15}$ The time-course of cerebral lactate increase during hypoxic-ischemic brain injury have been shown in animal models to have a biphasic course, that is, lactate levels rise as early as 3 hours, then returns to baseline after reoxygenation, and then rise again after 12 to 24 hours $^{19}$; hypothermia decrease this delayed rise. ${ }^{20}$ In addition, in the absence of hypoxia, hypoglycemia may lead to elevated lactate in the brain through astrocyte lactate production, as astrocytes only contain only the lactate dehydrogenase enzyme, which preferentially convert pyruvate to lactate. ${ }^{21}$

The objective of this case series was to document whether lactate levels may be used as a surrogate to monitor the hemodynamics of asphyxiated newborns treated with hypothermia and to review which inotropic support would be the most optimal for them. In this case series, the course over the first days of life of four asphyxiated newborns treated with hypothermia is presented, and lactate levels are discussed according to systemic blood pressure and inotropic support.

\section{Methods}

\section{Patients}

Four term asphyxiated newborns with significant episodes of hypotension were included in this case series. A diagnosis of hypotension was made clinically if the mean arterial blood pressure was less than the gestational age. ${ }^{22}$ All the newborns were treated with hypothermia, since they all met the following clinical criteria: (1) gestational age $\geq 36$ weeks and birth weight $\geq 1,800 \mathrm{~g}$; (2) evidence of fetal distress, for example, a history of acute perinatal event, cord $\mathrm{pH} \leq 7.0$ or base deficit $\leq-16 \mathrm{mEq} / \mathrm{L}$; (3) evidence of neonatal distress, such as an Apgar score $\leq 5$ at 10 minutes, postnatal blood gas $\mathrm{pH}$ obtained within the first hour of life $\leq 7.0$ or base deficit $\leq-16 \mathrm{mEq} / \mathrm{L}$, or a continued need for ventilation initiated at birth and continued for at least 10 minutes; and (4) evidence of moderate or severe encephalopathy determined by a physical examination and/or amplitude-integrated electroencephalogram. The four term asphyxiated newborns were admitted to the neonatal intensive care unit (NICU) within the first 6 hours of life and received whole-body cooling to an esophageal temperature of $33.5^{\circ} \mathrm{C}$ for 72 hours. After 72 hours, they were slowly rewarmed with a gradual increase of set blanket temperature of $0.5^{\circ} \mathrm{C} / \mathrm{h}$. The research protocol was approved by the institutional review board, and informed parental consent was obtained in all cases.

\section{Clinical Data}

Clinical data were collected for each patient, which included gestational age, birth weight, sex, Apgar scores, intubation, and chest compression at birth, presence of meconium at birth, arterial cord $\mathrm{pH}$, and initial infant blood gas $\mathrm{pH}$. The initial Sarnat score and background pattern of amplitudeintegrated electroencephalogram was assessed on 
admission. ${ }^{23,24}$ To assess the relation between serial serum lactate levels, blood pressure, and inotropic support, lactate levels were compared with the mean arterial blood pressure of the four newborns within the first 4 days of life. Lactate levels were monitored by regular blood gases (most often arterial, since an arterial line is usually inserted in these newborns). The mean arterial blood pressure was obtained from the flowsheets filled in by the nurses for each newborn every hour and/or when it was deemed abnormal. The mean arterial blood pressures were measured via an indwelling catheter or blood pressure cuffs. The introduction and changes of inotropic support medications were recorded. Any associated persistent pulmonary hypertension was diagnosed clinically when a persistent significant difference of $\geq 10$ was present between the preductal and postductal oxygen saturation levels ${ }^{1}$; an echocardiogram also was performed to confirm any suprasystemic pulmonary hypertension.

The outcome was recorded for each patient. The presence and extent of brain injury were evaluated with magnetic resonance imaging (MRI) scans performed around day 10 of life or by autopsy when the newborn did not survive up to day 10 of life. Each MRI study included T1- and T2-weighted imaging, and diffusion-weighted imaging. Neuroradiologists, who were blind to the clinical condition of the newborns, reviewed each imaging and scored it using a previously described MRI scoring system. ${ }^{25}$

\section{Statistical analysis}

After the detailed description of each of these four cases, we explored the relationship between time after birth, mean arterial blood pressure, and lactate levels by using all the different values of the four newborns. The association between time after birth and mean arterial blood pressure, time after birth and lactate levels, as well as mean arterial blood pressure and lactate levels were explored using Spearmen correlations. A $p$ value $<0.05$ was considered as statistically significant. All statistical analyses were performed using GraphPad Prism (GraphPad Software Inc., San Diego, CA).

\section{Results}

The clinical characteristics of the four newborns included in this case series are shown in -Table 1. They were all intubated soon after birth; two of the newborns required chest compression at birth. They all were treated with hypothermia. Newborn 4 , who had a hypotension refractory to treatments, died from the complications of neonatal encephalopathy; the other three newborns survived. Newborns 1 and 4 presented with seizures during hypothermia; newborn 1 was treated with phenobarbital, phenytoin, and topiramate, whereas newborn 4 only needed phenobarbital to get the seizures under control. Newborns 2 and 3 did not present any seizures during hypothermia. Newborn 3 did not develop brain injury, but all the others did, that is, newborn 2 developed basal ganglia injury, and newborns 1 and 4 developed near-total brain injury. None of the newborns presented with clinical signs of chorioamnionitis.
All four newborns presented hypotension and required boluses of normal saline, inotropic support, and hydrocortisone (doses of $1 \mathrm{mg} / \mathrm{kg}$ ). Cortisol level was measured in two newborns, before starting hydrocortisone treatment, that is, newborns 2 and 3 had a cortisol level of 92.3 and $112.6 \mathrm{nmol} /$ $\mathrm{L}$, respectively. All the newborns presented with associated pulmonary hypertension and required nitric oxide; in addition, newborn 3 was started on a milrinone drip.

The lactate levels in these newborns were not measured in the blood gas performed within the first hour of life, since the blood gas was done at the referring hospital before the transfer to our NICU. Measurements of lactate levels have started since the admission in our NICU. Lactate levels were high on admission, but initially decreased when blood pressure was adequate. However, after this first decrease, each of the newborns started to present lower blood pressure, and their lactate levels started to increase again (- Figs. 1-4).

In addition to boluses of normal saline, inotropic support and hydrocortisone was started for these newborns to improve their blood pressure. Dopamine and dobutamine were used with all four newborns and usually lead to an improvement in blood pressure, and a clearing of lactate levels. Epinephrine was also used with all these newborns at some time points during the hypothermia or the rewarming. The use of an epinephrine drip improved blood pressure, but was associated with an increase of lactate levels in all four patients (-Figs. 1-4); the lactate levels of newborns 1,2 , and 3 , improved once the epinephrine drip was discontinued. Vasopressin was used only in newborn 4 who presented with hypotension refractory to every inotropic support agent, and who eventually passed away (-Fig. 4).

While on inotropic support and before rewarming, newborn 1 presented with an episode of elevated blood pressure, which also coincided with an increase in lactate levels (-Fig. 1).

All the newborns presented these episodes of hypotension within the first 24 hours of life and then remained stable (except newborn 4) during the remaining part of the hypothermia treatment while on inotropic support. By the time of the rewarming, inotropic support was weaned or in the process to be weaned. However, in our three newborns who survived, rewarming was associated with hemodynamics perturbations (decrease in blood pressure and/or increase of lactate levels), that is, newborns 1 and 3 had episodes of lower blood pressure (-Figs. 1 and $\mathbf{3}$ ), and newborn 2 had an increase of lactate levels (-Fig. 2).

When plugging the data of all the patients to look at the relationship between time after birth, mean arterial blood pressure, and lactate levels, statistical analysis using Spearman correlations showed a weak but significant positive correlation between time after birth and mean arterial blood pressure ( $r=0.39, p<0.0001,293$ measurements) (-Fig. 5A). There was a significant moderate negative correlation between time after birth and lactate level ( $r=-0.58, p<0.0001,66$ measurements) (-Fig. 5A). There was a weak but significant negative correlation between mean arterial blood pressure and lactate level ( $r=-0.34, p=0.006,64$ measurements) (-Fig. 5B). 
Table 1 Clinical characteristics of the newborns

\begin{tabular}{|c|c|c|c|c|}
\hline Clinical data & Newborn 1 & Newborn 2 & Newborn 3 & Newborn 4 \\
\hline Gestational age (wks) & 39.86 & $34.71(?)$ & 39.57 & 39.00 \\
\hline Birth weight (g) & 3,980 & 4,050 & 3,670 & 2,800 \\
\hline Gender & Male & Male & Female & Female \\
\hline Apgar $1 \mathrm{~min}$ & 1 & 2 & 2 & 0 \\
\hline Apgar 5 min & 4 & 4 & 3 & 4 \\
\hline Apgar $10 \mathrm{~min}$ & 4 & 6 & 5 & 5 \\
\hline Intubation at birth & Yes & Yes & Yes & Yes \\
\hline Chest compression at birth & Yes & No & No & Yes \\
\hline Cord $\mathrm{pH}$ & 6.62 & 6.85 & 7.10 & 7.02 \\
\hline Gas $\mathrm{pH}$ in 1 st $\mathrm{h}$ of life & 6.69 & $\mathrm{n} / \mathrm{a}$ & 7.06 & 6.72 \\
\hline Lactate level $(\mathrm{mmol} / \mathrm{L})$ in $1 \mathrm{st} \mathrm{h}$ of life & $\mathrm{n} / \mathrm{a}$ & $\mathrm{n} / \mathrm{a}$ & $\mathrm{n} / \mathrm{a}$ & $\mathrm{n} / \mathrm{a}$ \\
\hline Initial Sarnat score & 3 & 2 & 2 & 3 \\
\hline Initial aEEG background pattern & Severe & Moderate & Severe & Severe \\
\hline Cooling & Yes & Yes & Yes & Yes \\
\hline Onset of hypothermia (h) & 3.65 & 5.68 & 4.77 & 5.15 \\
\hline Clinical chorioamnionitis & No & No & No & No \\
\hline Lactate level $(\mathrm{mmol} / \mathrm{L})$ on admission in the NICU & 9.4 & 12.4 & 5.9 & 24.0 \\
\hline Highest lactate level (mmol/L) during NICU stay & 12.9 & 17.0 & 11.7 & 26.0 \\
\hline Hypotension & Yes & Yes & Yes & Yes \\
\hline Bolus $\mathrm{NaCl}(10 \mathrm{~mL} / \mathrm{kg})$ & Yes & Yes & Yes & Yes \\
\hline Dopamine (maximal dose in $(\mu \mathrm{g} / \mathrm{kg} / \mathrm{min}))$ & 14 & 12 & 15 & 20 \\
\hline Epinephrine (maximal dose in $(\mu \mathrm{g} / \mathrm{kg} / \mathrm{min})$ ) & 0.10 & 0.10 & 0.05 & 0.25 \\
\hline Dobutamine (maximal dose in $(\mu \mathrm{g} / \mathrm{kg} / \mathrm{min})$ ) & 10 & 5 & 12 & 20 \\
\hline Vasopressin (maximal dose in (unit/kg/min)) & - & - & - & 0.003 \\
\hline Hydrocortisone & Yes & Yes & Yes & Yes \\
\hline Persistent pulmonary hypertension & Yes & Yes & Yes & Yes \\
\hline Nitric oxide & Yes & Yes & Yes & Yes \\
\hline Milrinone (maximal dose in $(\mu \mathrm{g} / \mathrm{kg} / \mathrm{min}))$ & - & - & 0.7 & - \\
\hline Outcome & Alive & Alive & Alive & Dead \\
\hline Seizures & Yes & No & No & Yes \\
\hline Brain injury & Near-total injury & Basal ganglia injury & No brain injury & Near-total injury \\
\hline
\end{tabular}

Abbreviations: aEEG, amplitude-integrated electroencephalogram; NICU, neonatal intensive care unit.

Note: The initial background pattern of the aEEG was assessed on admission and categorized into moderate or severe encephalopathy. ${ }^{23,24}$ Each magnetic resonance imaging (MRI) was scored using a previously described MRI scoring system. ${ }^{25}$

\section{Discussion}

In asphyxiated newborns, the initial lactate levels reflect the degree of the initial asphyxia event. This initial increase is due to an increase of lactic acid production secondary to the catecholamine stimulation of glycolysis and cellular hypoxia, as well as reduced hepatic clearance. ${ }^{26}$ However, after this initial increase, the lactate levels should slowly come back to normal. Delayed lactate clearance has been explained in animal models of asphyxia by a delayed hepatic clearance and an increased production of lactate via the stimulation of glycolysis and the sympathetic adrenal system, ${ }^{26}$ or by seizure activity. ${ }^{27}$ In addi- tion, Shah et $\mathrm{al}^{28}$ demonstrated that lactate levels took longer to normalize in asphyxiated newborns with moderate-to-severe neonatal encephalopathy, compared with newborns with mild neonatal encephalopathy. ${ }^{28}$ Animal studies have also shown that the worst histological evidence of brain injuries associated with the persistent elevation of lactate, which suggests that increased lactate levels may serve as a marker for ongoing tissue hypoxia. ${ }^{29}$ The current case series demonstrated that lactate level decrease over time in asphyxiated newborns during hypothermia treatment, but in some cases a secondary increase in lactate levels also might reflect a suboptimal hemodynamics status in these newborns, and may thus compromise further the brain 


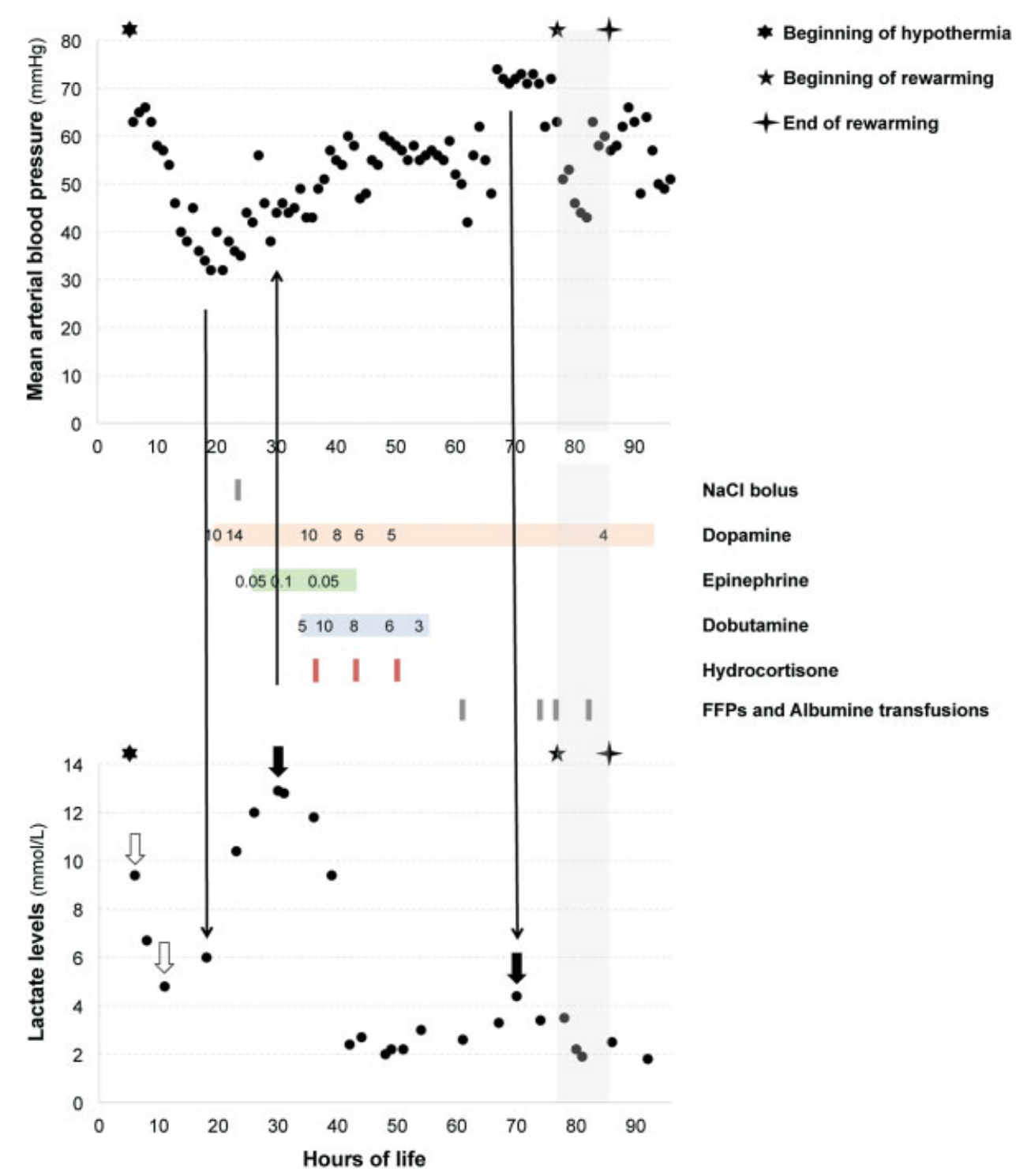

Fig. 1 Mean arterial blood pressure, lactate levels, and inotropic support for newborn 1 during the first 4 days of life. Dopamine, epinephrine, and dobutamine are expressed in $\mu \mathrm{g} / \mathrm{kg} / \mathrm{min}$. Lactate levels initially decreased. However, the newborn started to present lower blood pressure, and lactate levels started to increase again (arrow pointing down). He received a bolus of normal saline, and was started on dopamine. He was also started on epinephrine, since low blood pressure persisted, but his lactate levels increased higher (arrow pointing up) even when his blood pressure normalized. After switching the epinephrine for dobutamine and starting hydrocortisone, the blood pressure remained adequate, and lactate levels decreased. He later presented an episode of elevated blood pressure associated with a slight increase of his lactate level (arrow pointing down). The time of rewarming (gray rectangle) coincided with a decrease of blood pressure.

perfusion and possibly worsen brain injury. During hypothermia treatment, the four newborns presented an increase of lactate levels beyond the initial value recorded for each of them. In each of the described newborns, episodes of hypotension were associated with an increase of the lactate levels. There was a significant negative correlation between the lactate levels and the mean blood pressure values. This secondary increase of lactate levels probably indicates an ongoing anaerobic metabolism and possibly an ongoing tissue hypoxia. The four newborns also presented persistent pulmonary hypertension, which may in itself significantly impair brain oxygenation during the first days of life and may contribute to the development of brain injury in these newborns despite therapeutic hypothermia. ${ }^{30} \mathrm{At}$ the cellular level, systemic hypotension and suboptimal oxygenation lead to inhibition of the mitochondrial oxidative phos- phorylation, which immediately induce a depletion of glucose, glycogen, and adenosine triphosphate storage and elevation of lactate levels. ${ }^{14}$ Elevation of hydrogen ion as a result of adenosine triphosphate consumption and lactate production leads to acidosis, which inhibits glycolysis through inhibition of the phosphofructokinase enzyme. $^{16}$

Before the hypothermia era, treatment was only supportive for asphyxiated newborns with multiorgan failure in an effort to maintain as much as possible homeostasis and prevent ongoing brain injury. ${ }^{31}$ Strict monitoring and prompt correction were recommended for common complications, including blood pressure and oxygenation. ${ }^{31}$ Similar goals should be targeted now with ongoing hypothermia treatment to support the optimum effectiveness of this treatment. 


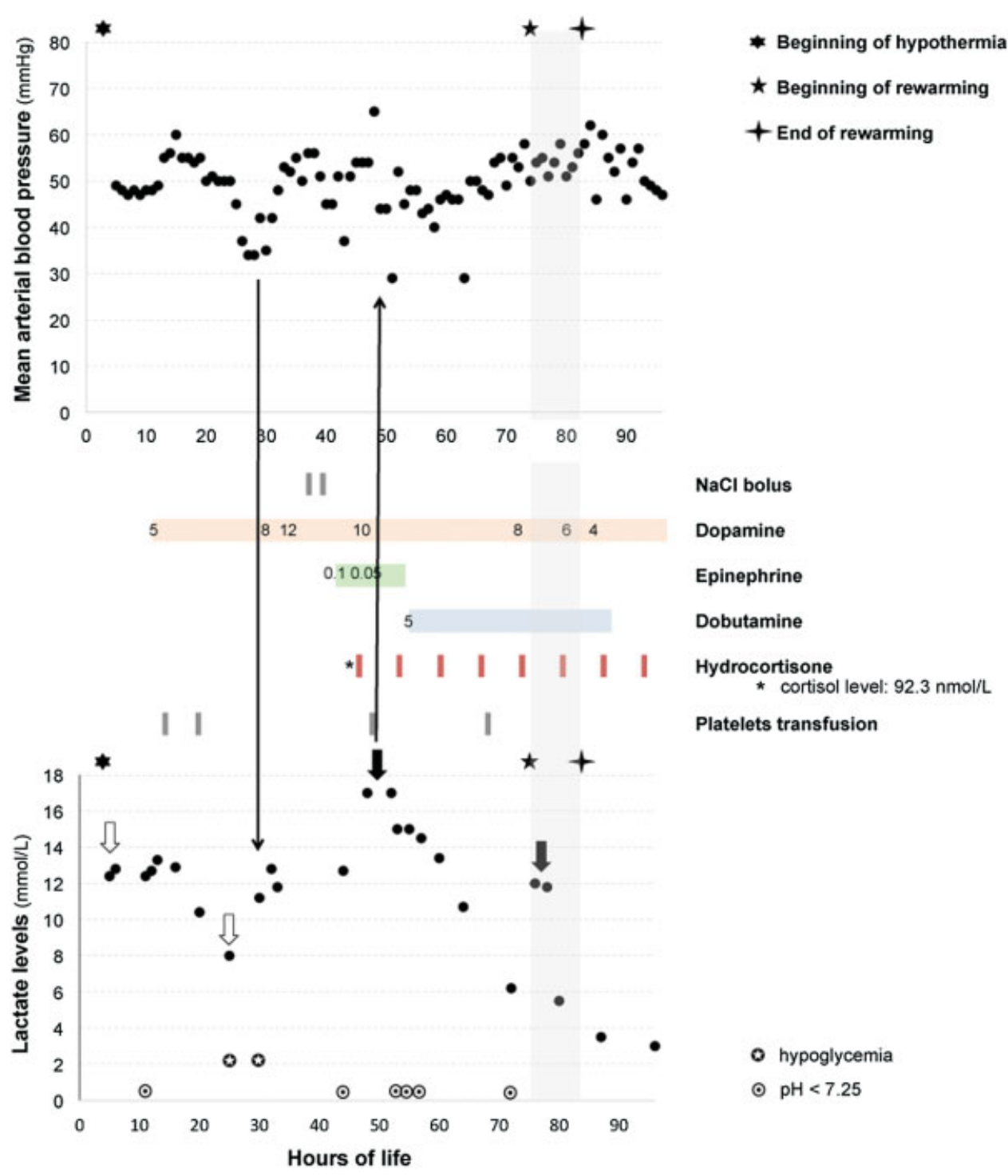

Fig. 2 Mean arterial blood pressure, lactate levels, and inotropic support for newborn 2 during the first 4 days of life. Dopamine, epinephrine, and dobutamine are expressed in $\mu \mathrm{g} / \mathrm{kg} / \mathrm{min}$. Lactate levels initially decreased. However, the newborn started to present lower blood pressure, and lactate levels started to increase again (arrow pointing down). He was started on dopamine. Since he presented persistent pulmonary hypertension and signs of right-to-left shunting with a significant difference between the pre- and postductal saturations, he received a bolus of normal saline and was also started on epinephrine to maintain an adequate blood pressure, but his lactate levels increased even higher (arrow pointing up). After switching the epinephrine for dobutamine and starting hydrocortisone, his blood pressure remained adequate and his lactate level decreased. The time of rewarming (gray rectangle) coincided with an increase of his lactate level.

The management of suboptimal hemodynamics in asphyxiated newborns treated with hypothermia requires exogenous catecholamine support. However, randomized controlled trials are lacking with regard to recommending which inotropic agents are the most optimal for this specific group of newborns. ${ }^{32}$ Although dopamine has been shown to be effective in treating systemic hypotension in five randomized controlled trials involving newborns, ${ }^{33}$ all these trials were conducted with premature newborns, who were born at less than 36 weeks. Thus, these studies are difficult to extrapolate to near-term and term asphyxiated newborns due to the developmental and pathophysiological changes that occur at various gestational ages and following asphyxia. For example, the expression of adrenoceptors in the heart is decreased in preterm newborns compared with term newborns. ${ }^{34,35}$ In addition, during asphyxia, the expression of adrenoceptors increases in the pulmonary circulation. ${ }^{36}$ In our case series, dopamine and dobutamine enabled the clearance of the lactate levels and improved blood pressure (except for newborn 4 who had refractory hypotension), whereas the epinephrine drip consistently worsened the increase of lactate levels in these newborns. The lactate levels improved in newborns 1,2 , and 3 once the epinephrine drip was discontinued. The effects of dopamine, epinephrine, dobutamine, and milrinone have been examined in an animal model of asphyxia; all of them increased the mean arterial blood pressure, stroke volume, and cardiac output without worsening pulmonary hypertension. ${ }^{37}$ Furthermore, even low doses of dopamine have been shown to increase systolic blood pressure and, to a lesser extent, diastolic blood pressure in severely asphyxiated newborns. ${ }^{38}$ However, 
e54 Lactate Levels during Hypothermia in Asphyxiated Newborns Al Balushi et al.

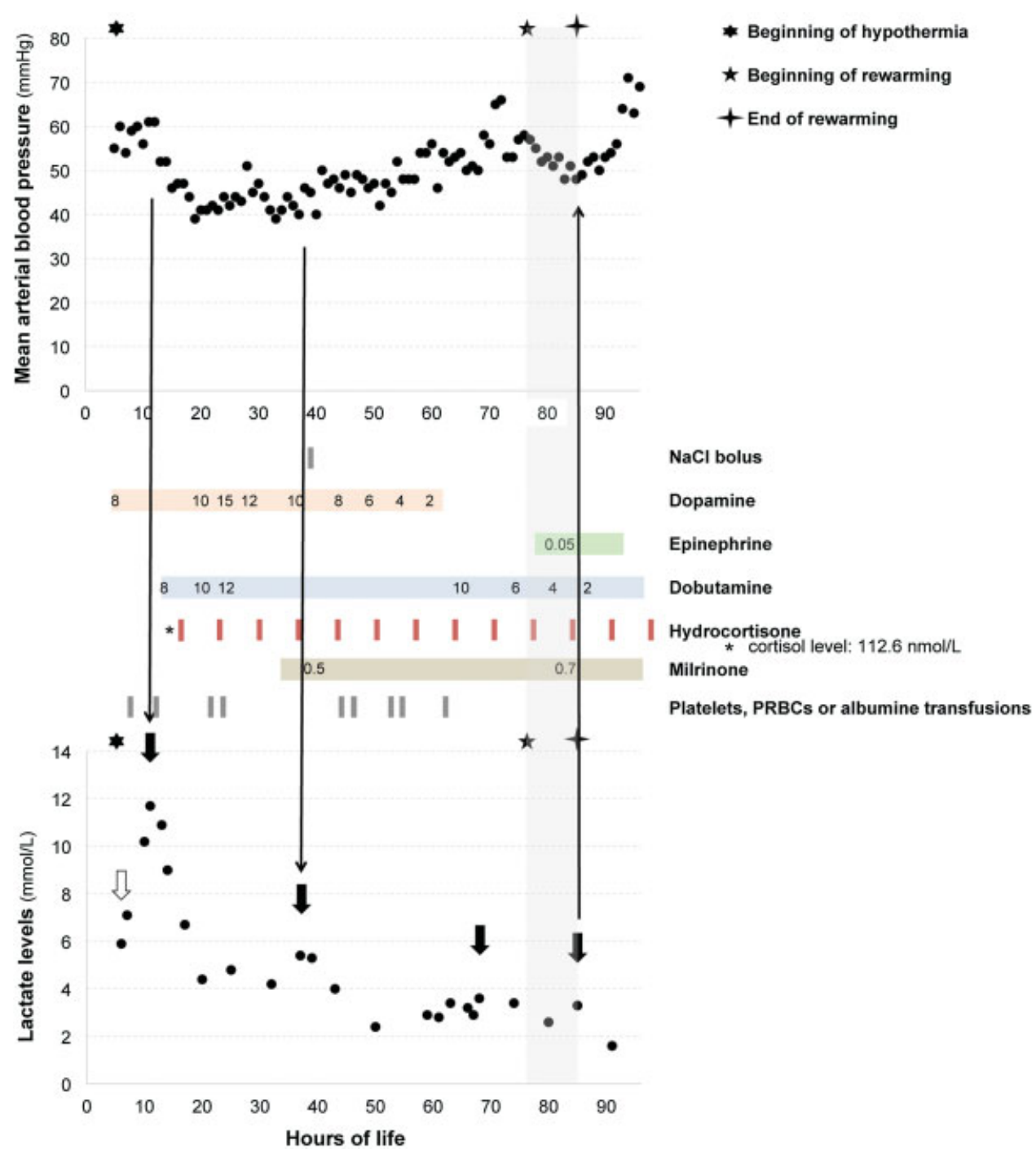

Fig. 3 Mean arterial blood pressure, lactate levels, and inotropic support for newborn 3 during the first 4 days of life. Dopamine, epinephrine, dobutamine, and milrinone are expressed in $\mu \mathrm{g} / \mathrm{kg} / \mathrm{min}$. Lactate levels were at 5.9 on admission to the neonatal intensive care unit. However, the newborn started to present lower blood pressure, and lactate levels started to increase again (arrows pointing down). She was started on dopamine, dobutamine, and also hydrocortisone. As she presented persistent pulmonary hypertension, she was started on milrinone in addition to nitric oxide. Her blood pressure corrected and her lactate level decreased. The time of rewarming (gray rectangle) coincided with a decrease of blood pressure. At that time, an epinephrine drip was started, but her lactate level increased higher (arrow pointing up); the epinephrine drip was discontinued and lactate levels decreased again.

dopamine may also increase pulmonary vascular resistance, ${ }^{39}$ although this finding was not consistent across the different studies. ${ }^{38}$ One additional potential benefit of dopamine was its ability to prevent loss of cerebral blood flow autoregulation in an animal model of traumatic brain injury, ${ }^{40}$ in contrast to dobutamine, which has been shown to have no effects on cerebral hemodynamics. ${ }^{41}$ Alternatively, studies suggest that epinephrine may have a more favorable hemodynamic profile, since it improves systemic blood pressure without exacerbating pulmonary hypertension, and since it has been shown in one study to have vasodilatory effects on the pulmonary vasculature. ${ }^{37}$ However, epinephrine also has been shown to increase lactic acid production in some patients, probably via stimulation of $\beta$-receptors rather than via increased anaerobic metabolism, ${ }^{42}$ which makes it difficult to differentiate with regard to the newborns of our case series whether an increase in serum lactate was due to ongoing tissue hypoxia or an epinephrine-induced stimulation of $\beta$ receptors. In addition, during hypothermia, epinephrine may fail to improve cardiac output due to a downregulation of the $\beta$-adrenoreceptors. ${ }^{43}$ In surgical patients with postcardiopulmonary bypass, this kind of increase of lactate levels has not been related to reduced tissue perfusion, ${ }^{42}$ but nevertheless has been linked to an increase in whole-body blood flow and a decrease in wholebody oxygen extraction, ${ }^{42}$ which could be detrimental in asphyxiated newborns, for whom hypothermia treatment is supposed to prevent secondary brain injury by reducing metabolic demand and perfusion.

Rewarming is another time period that can lead to disturbances in cerebral blood flow autoregulation by increasing metabolic demand. ${ }^{44}$ In the three newborns who survived, rewarming was associated with hemodynamics perturbations. Peripheral vasodilatation typically occurs during rewarming, which increases the intravascular blood volume 


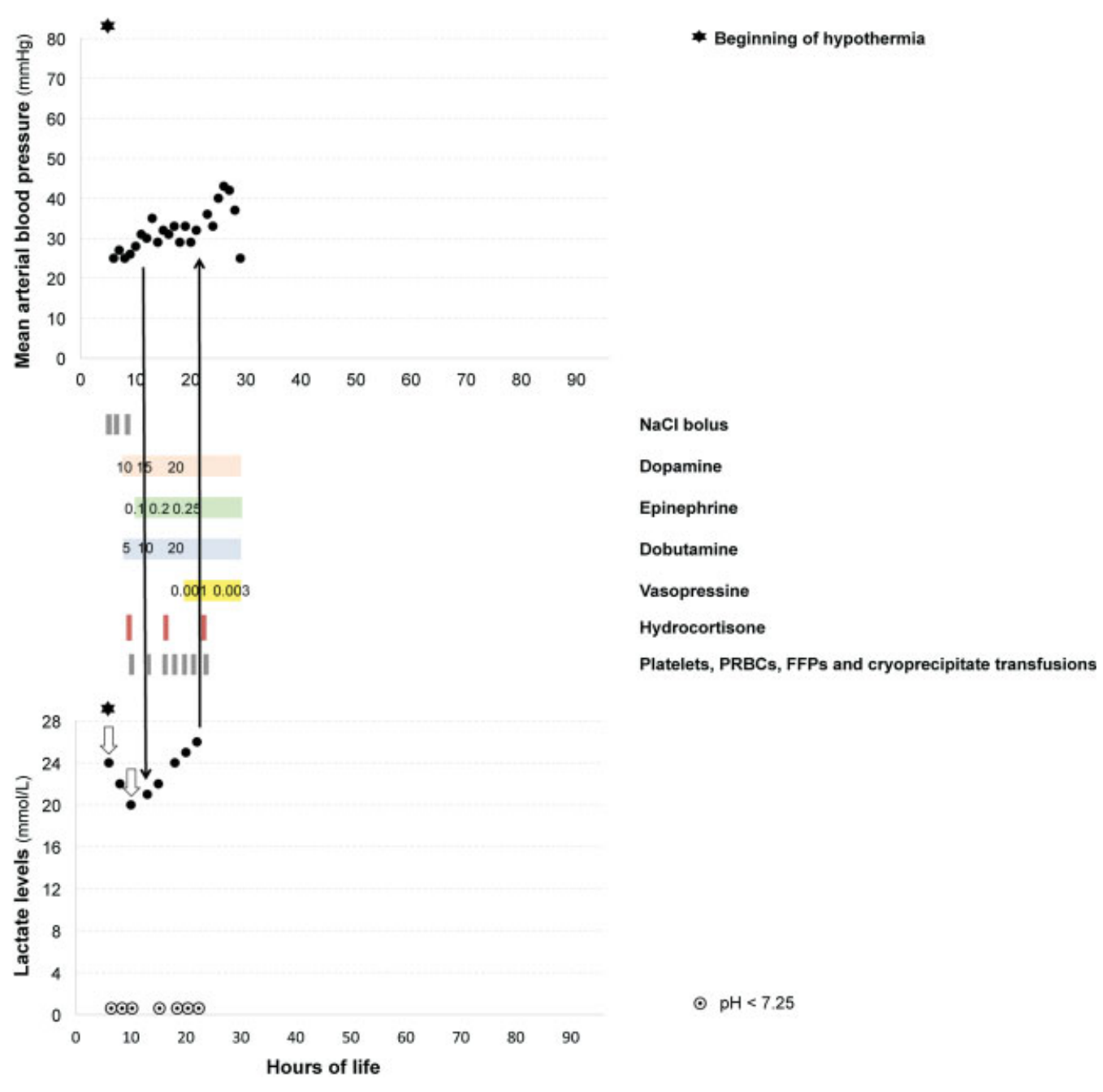

Fig. 4 Mean arterial blood pressure, lactate levels, and inotropic support for newborn 4 during the first 4 days of life. Dopamine, epinephrine and dobutamine are expressed in $\mu \mathrm{g} / \mathrm{kg} / \mathrm{min}$. Doses for vasopressin are expressed in units $/ \mathrm{kg} / \mathrm{min}$. Lactate levels initially decreased, but this newborn quickly presented with severe hypotension. Lactate levels started to increase again (arrows pointing down). She received boluses of normal saline, was started on dopamine, dobutamine, and hydrocortisone. An epinephrine drip was added, and her lactate level continued to increase. A vasopressin drip was added, and her blood pressure improved transiently, but the lactate levels continued to increase. Shortly after, the blood pressure decreased again and became refractory to all these treatments. Eventually, she died from the complications of neonatal encephalopathy.
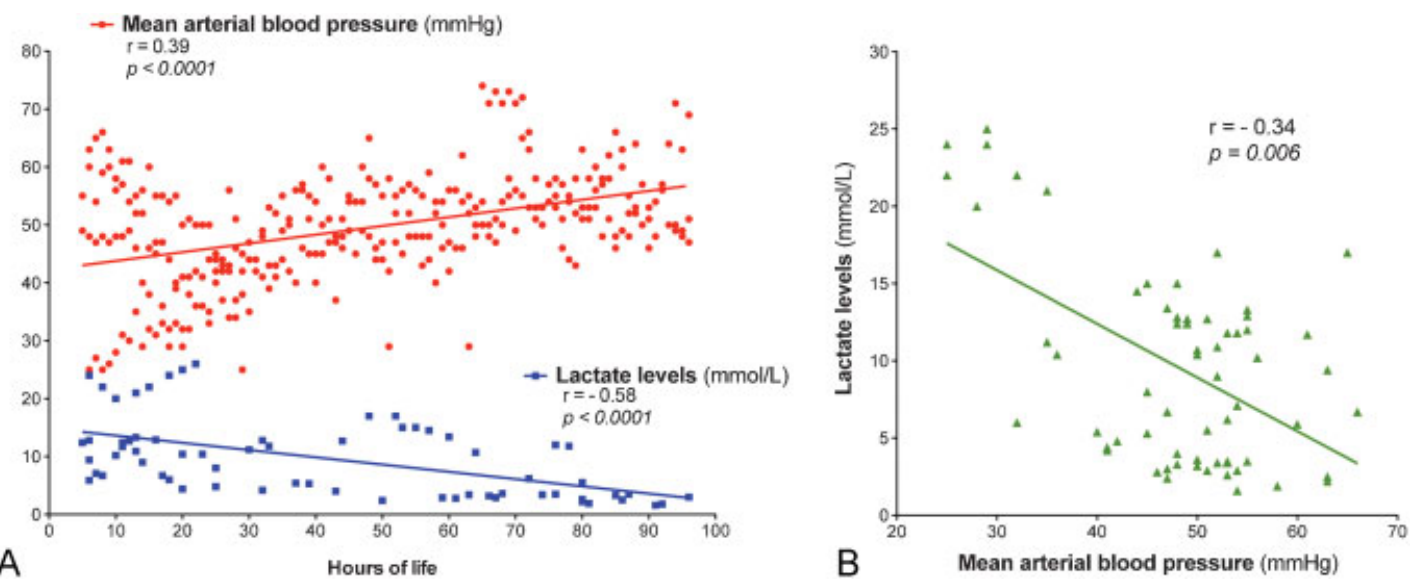

Fig. 5 Relationship between time after birth, mean arterial blood pressure, and lactate levels. (A) Comparison between time after birth (h), mean arterial blood pressure $(\mathrm{mm} \mathrm{Hg})$, and lactate levels $(\mathrm{mmol} / \mathrm{L})$. Mean blood pressure increased over time $(r=0.39, p<0.0001)$, whereas lactate levels decreased over time $(r=-0.58, p<0.0001)$. (B) Comparison between mean arterial blood pressure $(\mathrm{mm} \mathrm{Hg})$ and lactate levels (mmol/L). There was a negative correlation between mean arterial blood pressure and lactate levels $(r=-0.34, p=0.006)$. 
and often leads to hypotension. ${ }^{45}$ During rewarming, blood pressure drops and heart rate increases. ${ }^{10,46}$ Cardiac output was higher during rewarming than during cooling. ${ }^{47} \mathrm{Re}-$ warming also is thought to cause a mismatch between oxygen delivery and consumption. ${ }^{48,49}$ Hypotension during rewarming has been described as a risk factor for severe brain injury and intraventricular hemorrhage. ${ }^{46,50}$ In addition, rapid rate of rewarming has been incriminated as an inducing factor of brain injury, lung injury, and hypotension in various pathological conditions such as hemorrhagic shock, traumatic brain injury, and sepsis. ${ }^{51-53}$ Clinical trials in asphyxiated newborns typically use a rewarming rate of $0.5^{\circ} \mathrm{C} / \mathrm{h}$; however, lower rate of rewarming have also been suggested to avoid any of these potential complications, which may further worsen brain injury in this vulnerable population. ${ }^{54}$

Optimally, all these asphyxiated newborns treated with hypothermia should have serial functional echocardiograms during hypothermia and rewarming to assess in real-time their cardiovascular performance and systematic hemodynamics $^{55}$ and to adjust more specifically their need for inotropic support. However, in many institutions, neonatologists still are not trained to perform these functional echocardiograms, and access to a regular echocardiography remains difficult to obtain in a timely manner, and very often only provides one-time information on cardiac anatomy and cardiovascular performance. ${ }^{55}$ Our case series suggests that a close monitoring of lactate levels may be a surrogate for the monitoring of hemodynamics in these newborns. In our experience, neonatologists usually carefully look at the first values of lactic acid for prognostication, but then often do not follow closely the evolution of these lactate levels. Previous studies on the hemodynamics of asphyxiated newborns treated with hypothermia did not perform routine measurements of lactate levels. ${ }^{5}$ Until now, studies of the cardiac function of asphyxiated newborns have evaluated the use of troponin and repeated echocardiograms to monitor cardiac injury, ${ }^{56}$ but they did not perform routine measurements of lactate levels to monitor hemodynamics. ${ }^{5}$

These four newborns may not be representative of most of the asphyxiated newborns treated with hypothermia, since they were severely asphyxiated newborns with multiorgan failure, and thus this case series is not able to offer definitive conclusions about which inotropic support is the most optimal for asphyxiated newborns. However, this case series raises the concern that hypotension episodes may impact brain injury in asphyxiated newborns. A consensus about which inotropic agent is the best for these newborns does not exist. Future randomized studies such as the ones performed with premature newborns ${ }^{33}$ should be performed with asphyxiated newborns treated with hypothermia to determine whether maintaining hemodynamic stability during hypothermia and rewarming improves the long-term neurodevelopmental outcome and to determine which inotropic support is optimal for these newborns, so that management guidelines based on evidence can be developed and benefit the management of these newborns. In the meantime, these secondary increases of lactate levels as described in the newborns in our case series should probably be avoided to support an optimized hemodynamics for newborns already at risk of brain injury.

\section{Conclusions}

In conclusion, when functional echocardiography is not readily available in the NICU, lactate levels during the first 4 days of life should be carefully monitored and may serve as a surrogate marker for the suboptimal hemodynamic status of term asphyxiated newborns treated with hypothermia, for whom the maintenance of homeostasis during hypothermia and rewarming is of the utmost importance to alleviate brain injury. Further prospective randomized studies are warranted to determine the impact of hypotension on mortality and brain injury and to determine the best inotropic agents for these newborns.

\section{Acknowledgments}

The authors would like to thank the families and their newborns for participating in this study. They also thank Mr. Wayne Ross Egers for his professional English correction of the article. Pia Wintermark receives research grant funding from the FRSQ Clinical Research Scholar Career Award Junior 1, and a New Investigator Research Grant from the SickKids Foundation and the CIHR Institute of Human Development, Child and Youth Health.

\section{References}

1 Jacobs SE, Berg M, Hunt R, Tarnow-Mordi WO, Inder TE, Davis PG. Cooling for newborns with hypoxic ischaemic encephalopathy. Cochrane Database Syst Rev 2013;1:CD003311

2 Cavallaro G, Filippi L, Raffaeli G, et al. Heart rate and arterial pressure changes during whole-body deep hypothermia. ISRN Pediatr 2013;2013:140213

3 Walther FJ, Siassi B, Ramadan NA, Wu PY. Cardiac output in newborn infants with transient myocardial dysfunction. J Pediatr 1985;107(5):781-785

4 Leone TA, Finer NN. Shock: a common consequence of neonatal asphyxia. J Pediatr 2011;158(2, Suppl):e9-e12

5 Gebauer CM, Knuepfer M, Robel-Tillig E, Pulzer F, Vogtmann C. Hemodynamics among neonates with hypoxic-ischemic encephalopathy during whole-body hypothermia and passive rewarming. Pediatrics 2006;117(3):843-850

6 Wood T, Thoresen M. Physiological responses to hypothermia. Semin Fetal Neonatal Med 2015;20(2):87-96

7 Hsu CY, Huang CH, Chang WT, et al. Cardioprotective effect of therapeutic hypothermia for postresuscitation myocardial dysfunction. Shock 2009;32(2):210-216

8 Kobayashi M, Godin D, Nadeau R. Sinus node responses to perfusion pressure changes, ischaemia and hypothermia in the isolated blood-perfused dog atrium. Cardiovasc Res 1985; 19(1):20-26

9 Chernow B, Lake CR, Zaritsky A, et al. Sympathetic nervous system "switch off" with severe hypothermia. Crit Care Med 1983;11(9): 677-680

10 Zhou J, Poloyac SM. The effect of therapeutic hypothermia on drug metabolism and response: cellular mechanisms to organ function. Expert Opin Drug Metab Toxicol 2011;7(7):803-816

11 Han YS, Tveita T, Kondratiev TV, Prakash YS, Sieck GC. Changes in cardiovascular beta-adrenoceptor responses during hypothermia. Cryobiology 2008;57(3):246-250

12 Rios DR, Moffett BS, Kaiser JR. Trends in pharmacotherapy for neonatal hypotension. J Pediatr 2014;165(4):697-701.e1 
13 Rosenberg AA. Regulation of cerebral blood flow after asphyxia in neonatal lambs. Stroke 1988;19(2):239-244

14 Fernandez F, Verdu A, Quero J, et al. Cerebrospinal fluid lactate levels in term infants with perinatal hypoxia. Pediatr Neurol 1986; 2(1):39-42

15 Leth H, Toft PB, Peitersen B, Lou HC, Henriksen O. Use of brain lactate levels to predict outcome after perinatal asphyxia. Acta Paediatr 1996;85(7):859-864

16 Vannucci RC, Brucklacher RM, Vannucci SJ. Glycolysis and perinatal hypoxic-ischemic brain damage. Dev Neurosci 2005;27(2-4): 185-190

17 Vannucci RC, Yager JY, Vannucci SJ. Cerebral glucose and energy utilization during the evolution of hypoxic-ischemic brain damage in the immature rat. J Cereb Blood Flow Metab 1994;14(2): 279-288

18 Larach DB, Kofke WA, Le Roux P. Potential non-hypoxic/ischemic causes of increased cerebral interstitial fluid lactate/pyruvate ratio: a review of available literature. Neurocrit Care 2011; 15(3):609-622

19 Penrice J, Lorek A, Cady EB, et al. Proton magnetic resonance spectroscopy of the brain during acute hypoxia-ischemia and delayed cerebral energy failure in the newborn piglet. Pediatr Res 1997;41(6):795-802

20 Amess PN, Penrice J, Cady EB, et al. Mild hypothermia after severe transient hypoxia-ischemia reduces the delayed rise in cerebral lactate in the newborn piglet. Pediatr Res 1997;41(6): 803-808

21 Abi-Saab WM, Maggs DG, Jones T, et al. Striking differences in glucose and lactate levels between brain extracellular fluid and plasma in conscious human subjects: effects of hyperglycemia and hypoglycemia. J Cereb Blood Flow Metab 2002;22(3): 271-279

22 Farrugia R, Rojas H, Rabe H. Diagnosis and management of hypotension in neonates. Future Cardiol 2013;9(5):669-679

23 al Naqeeb N, Edwards AD, Cowan FM, Azzopardi D. Assessment of neonatal encephalopathy by amplitude-integrated electroencephalography. Pediatrics 1999;103(6 Pt 1):1263-1271

24 Gluckman PD, Wyatt JS, Azzopardi D, et al. Selective head cooling with mild systemic hypothermia after neonatal encephalopathy: multicentre randomised trial. Lancet 2005; 365(9460):663-670

25 Barkovich AJ, Hajnal BL, Vigneron D, et al. Prediction of neuromotor outcome in perinatal asphyxia: evaluation of MR scoring systems. AJNR Am J Neuroradiol 1998;19(1):143-149

26 Stanley WC, Mazer CD, Neese RA, et al. Increased lactate appearance and reduced clearance during hypoxia in dogs. Horm Metab Res 1990;22(9):478-484

27 Murray DM, Boylan GB, Fitzgerald AP, Ryan CA, Murphy BP, Connolly S. Persistent lactic acidosis in neonatal hypoxic-ischaemic encephalopathy correlates with EEG grade and electrographic seizure burden. Arch Dis Child Fetal Neonatal Ed 2008;93(3): F183-F186

28 Shah S, Tracy M, Smyth J. Postnatal lactate as an early predictor of short-term outcome after intrapartum asphyxia. J Perinatol 2004; 24(1):16-20

29 Rey-Santano C, Mielgo VE, Gastiasoro E, et al. Early cerebral hemodynamic, metabolic, and histological changes in hypoxicischemic fetal lambs during postnatal life. Front Neurosci 2011; 5:111

30 Lapointe A, Barrington KJ. Pulmonary hypertension and the asphyxiated newborn. J Pediatr 2011;158(2, Suppl):e19-e24

31 Agarwal R, Jain A, Deorari AK, Paul VK. Post-resuscitation management of asphyxiated neonates. Indian J Pediatr 2008;75(2): $175-180$

32 Hunt R, Osborn D. Dopamine for prevention of morbidity and mortality in term newborn infants with suspected perinatal asphyxia. Cochrane Database Syst Rev 2002;3(3):CD003484
33 Subhedar NV, Shaw NJ. Dopamine versus dobutamine for hypotensive preterm infants. Cochrane Database Syst Rev 2003;3(3): CD001242

34 Kim MY, Finch AM, Lumbers ER, et al. Expression of adrenoceptor subtypes in preterm piglet heart is different to term heart. PLoS ONE 2014;9(3):e92167

35 Michikata K, Sameshima H, Sumiyoshi K, Kodama Y, Kaneko M, Ikenoue T. Developmental changes in catecholamine requirement, volume load and corticosteroid supplementation in premature infants born at 22 to 28 weeks of gestation. Early Hum Dev 2010; 86(7):401-405

36 Lock JE, Olley PM, Coceani F. Enhanced beta-adrenergic-receptor responsiveness in hypoxic neonatal pulmonary circulation. Am J Physiol 1981;240(5):H697-H703

37 Joynt C, Bigam DL, Charrois G, Jewell LD, Korbutt G, Cheung PY. Milrinone, dobutamine or epinephrine use in asphyxiated newborn pigs resuscitated with $100 \%$ oxygen. Intensive Care Med 2010;36(6):1058-1066

38 DiSessa TG, Leitner M, Ti CC, Gluck L, Coen R, Friedman WF. The cardiovascular effects of dopamine in the severely asphyxiated neonate. J Pediatr 1981;99(5):772-776

39 Drummond WH, Webb IB, Purcell KA. Cardiopulmonary response to dopamine in chronically catheterized neonatal lambs. Pediatr Pharmacol (New York) 1981;1(4):347-356

40 Armstead WM, Riley J, Vavilala MS. Dopamine prevents impairment of autoregulation after traumatic brain injury in the newborn pig through inhibition of Up-regulation of endothelin-1 and extracellular signal-regulated kinase mitogen-activated protein kinase. Pediatr Crit Care Med 2013;14(2):e103-e111

41 Kawamura S, Yasui N. Effects of dobutamine on brain surface microvessels in rats. Neurol Med Chir (Tokyo) 1998;38(3): 137-141, discussion 141-142

42 Totaro RJ, Raper RF. Epinephrine-induced lactic acidosis following cardiopulmonary bypass. Crit Care Med 1997;25(10):1693-1699

43 Kondratiev TV, Myhre ES, Simonsen O, Nymark TB, Tveita T. Cardiovascular effects of epinephrine during rewarming from hypothermia in an intact animal model. J Appl Physiol (1985) 2006;100(2):457-464

44 Chalak LF, Tarumi T, Zhang R. The "neurovascular unit approach" to evaluate mechanisms of dysfunctional autoregulation in asphyxiated newborns in the era of hypothermia therapy. Early Hum Dev 2014;90(10):687-694

45 Chakkarapani E, Thoresen M. Use of hypothermia in the asphyxiated infant. Perinatology 2010;3:20-29

46 Howlett JA, Northington FJ, Gilmore MM, et al. Cerebrovascular autoregulation and neurologic injury in neonatal hypoxic-ischemic encephalopathy. Pediatr Res 2013;74(5):525-535

47 Demirgan S, Erkalp K, Sevdi MS, et al. Cardiac condition during cooling and rewarming periods of therapeutic hypothermia after cardiopulmonary resuscitation. BMC Anesthesiol 2014; $14: 78$

48 Schwab S, Schwarz S, Spranger M, Keller E, Bertram M, Hacke W. Moderate hypothermia in the treatment of patients with severe middle cerebral artery infarction. Stroke 1998;29(12): 2461-2466

49 Enomoto S, Hindman BJ, Dexter F, Smith T, Cutkomp J. Rapid rewarming causes an increase in the cerebral metabolic rate for oxygen that is temporarily unmatched by cerebral blood flow. A study during cardiopulmonary bypass in rabbits. Anesthesiology 1996;84(6):1392-1400

50 Al Yazidi G, Srour M, Wintermark P. Risk factors for intraventricular hemorrhage in term asphyxiated newborns treated with hypothermia. Pediatr Neurol 2014;50(6):630-635

51 Alam HB, Rhee P, Honma K, et al. Does the rate of rewarming from profound hypothermic arrest influence the outcome in a swine model of lethal hemorrhage? J Trauma 2006;60(1): 134-146 
Al Balushi et al.

52 Lavinio A, Timofeev I, Nortje J, et al. Cerebrovascular reactivity during hypothermia and rewarming. Br J Anaesth 2007;99(2): 237-244

53 Maxwell WL, Watson A, Queen R, et al. Slow, medium, or fast rewarming following post-traumatic hypothermia therapy? An ultrastructural perspective. J Neurotrauma 2005;22(8):873-884

54 Laptook A, Tyson J, Shankaran S, et al; National Institute of Child Health and Human Development Neonatal Research Network.
Elevated temperature after hypoxic-ischemic encephalopathy: risk factor for adverse outcomes. Pediatrics 2008;122(3):491-499

55 El-Khuffash AF, McNamara PJ. Neonatologist-performed functional echocardiography in the neonatal intensive care unit. Semin Fetal Neonatal Med 2011;16(1):50-60

56 Costa S, Zecca E, De Rosa G, et al. Is serum troponin T a useful marker of myocardial damage in newborn infants with perinatal asphyxia? Acta Paediatr 2007;96(2):181-184 